\title{
Aronia Melanocarpa Polysaccharide Ameliotates Inflammation and Aging in Mice by Modulating AMPK/SIRT1/NF-KB Signaling Pathway and Gut Microbiota
}

\author{
Yingchun Zhao \\ Jilin Agricultural University \\ Xinglong Liu \\ Jilin Agricultural University \\ Yinan Zheng \\ Jilin Agricultural University \\ Wencong Liu ( $\sim$ jwlw6803@126.com ) \\ Jilin Agricultural University \\ Chuanbo Ding \\ Jilin Agricultural University
}

\section{Research Article}

Keywords: Aronia melanocarpa, signaling, AMP, D-galactose

Posted Date: August 4th, 2021

DOI: https://doi.org/10.21203/rs.3.rs-735001/v1

License: (c) (1) This work is licensed under a Creative Commons Attribution 4.0 International License.

Read Full License 


\title{
Aronia melanocarpa polysaccharide ameliotates inflammation and aging in mice by modulating AMPK/SIRT1/NF-KB signaling pathway and gut microbiota
}

\author{
Yingchun Zhao ${ }^{1,+}$, Xinglong Liu ${ }^{1,+}$, Yinan Zheng ${ }^{1,2,+}$, Wencong Liu ${ }^{1,2,{ }^{*}}$ and Chuanbo Ding ${ }^{1, *}$ \\ ${ }^{1}$ College of Chinese Medicinal Materials, Jilin Agricultural University, Changchun130118, China; \\ ${ }^{2}$ National \& Local Joint Engineering Research Center for Ginseng Breeding and Development, Changchun130118, \\ China. \\ *corresponding. jwlw6803@126.com; chuanboding0506@163.com. \\ +these authors contributed equally to this work.
}

\begin{abstract}
Aronia melanocarpa, as a natural medicinal plant, has been widely proved to have a variety of biological activities used as a novel food and medicine. Aronia melanocarpa polysaccharide (AMP) is the main component of the Aronia melanocarpa. The purpose of this research is to evaluate the delay and protection of AMP on aging mice by D-galactose (D-gal) induced, and to explore the effect of supplementing AMP on the metabolism of the intestinal flora of aging organisms.The aging model was established by intraperitoneal injection of D-gal $(200 \mathrm{mg} / \mathrm{kg}$ to $1000 \mathrm{mg} / \mathrm{kg})$ once per 3 days for 12 weeks, and administer AMP (100 and $200 \mathrm{mg} / \mathrm{kg}$ ) were given daily by oral gavage after 6 weeks of D-Gal-induced.The results showed that AMP treatment significantly improved the spatial learning and memory impairment of aging mice through the eight-arm maze test, and H\&E staining provedthat AMP significantly reversed brain tissue pathological damage and structural disorders.AMP alleviated inflammation and oxidative stress injury in aging brain tissue by regulating AMPK/SIRT1/NF- $\mathrm{B}$ and Nrf2/HO-1 signaling pathways. Particularly, AMP reduced brain cell apoptosis and neurological deficits by activating $\mathrm{PI} 3 \mathrm{~K} / \mathrm{AKT} / \mathrm{mTOR}$ signaling pathway and its downstream apoptotic protein family.Importantly, 16S rDNA analysis indicated the AMP treatment significantly redarded the aging process by improving the composition of intestinal flora and abundance of beneficial bacteria. In summary, this study found that AMP delayed brain aging in mice by inhibiting inflammatory and regulating intestinal microbes, which providing the possibility for the amelioration and treatment of aging and related metabolic diseases.
\end{abstract}

Abbreviations: AMP, Aronia melanocarpa polysaccharide; AMPK, Adenosine 5'-monophosphate (AMP)-activated protein kinase; SIRT1, Sirtuin 1; NF-кB, nuclear factor kappa-B; Nrf2, Nuclear related factor-2; HO-1, Heme oxygenase-1; PI3K, Phosphatidylinositol 3-kinase; AKT, Protein kinase B; mTOR, Mammalian rapamycin target protein; H\&E, Hematoxylin and Eosin; Fuc, fucose; Rha, rhamnose; Ara, arabinose; Gal, galactose; Glc, Glucose; Xyl, xylose; Man, mannose; Gal-UA, Galacturonic acid; Glc-UA, Glucuronic acid; Man-UA, Mannuronic acid; AChE, Acetyl cholinesterase; GSH, L-Glutathione; SOD, Superoxide Dismutase; CAT, Catalase;GSDMD, Gasdermin D; IL-1 $\beta$, Recombinant Rat IL-1 $\beta$; Bcl-2, B celllymphoma-2; Bax, B-associated X.

\section{Introduction}

Aging, as a natural process of life, was a degenerative disease of the function of living organisms and related nerves ${ }^{1}$. As an inevitable physiological process, aging would gradually lose its body functions, resulted in many diseases related to aging including diabetes, cognitive impairment, cancer, liver injury, Parkinson's disease and atherosclerosis, etc ${ }^{2,3}$. Both developed and developing countries are all exposed to aging society due to scientific and technological advancement. Therefore, delaying aging has become a worldwide focus on attention to various fields ${ }^{4}$. The Anti-aging effect mainly inhibiting telomere shortening, resisting lipid peroxidation, scavenging free radicals, regulating immune endocrine, reducing DNA damages and autophagy, etc ${ }^{5-9}$. AMPK (adenosine 5'-monophosphate (AMP) activated protein kinase) is an AMP-dependent protein kinase. It is a key molecule in regulation of biological energy metabolism and plays an important role in regulating cell growth, proliferation, survival and energy metabolism ${ }^{10-12}$. AMPK is involved in regulating a series of age-related signaling pathways such as SIRT1, NF- $\kappa$ B and p53 which play a role in the regulation of mammalian cell senescence ${ }^{13}$. Silencing message 
regulator 2-related enzyme 1(SIRTUIN 1) is a class of $\mathrm{NAD}^{+}$dependent histone deacetylases widely found in living bodies. SIRT1 is widely involved in cell survival, apoptosis, stress resistance, inflammation and other physiological activation. The activation of SIRT1 is an important reason for the extension of biological life span, It affects the anti-stress ability of cells by directly regulating p53 and NF- $\kappa B$ signaling pathways ${ }^{14,15}$. The regulatory function of AMPK in energy metabolism and the direct or indirect regulation of the above signaling pathways are important, so it is necessary to study the relationship between AMPK and the age-related signaling pathways to find the possible mechanisms of aging.

Aronia melanocarpaderives from North America, and it is introduced and cultivated in Northeast China in large-scale presently ${ }^{16}$. In September 2018, Aronia melanocarpa was approved to be a novel food by the National Health Council of China. It is mainly used for production of fruit juices, jams, sauces, fruit teas, dietary supplements and wines. While, it is also used as natural source of food coloring anti-hypertensive and anti-atherosclerotic drug in Russia and Eastern European countries ${ }^{17-20}$. Aronia melanocarpa is rich in flavonoids, polyphenols, polysaccharides, organic acids, dietary fiber and other nutrients. Polysaccharides are proved to beactive in anti-tumor, hepatoprotective, anti-inflammatory, anti-viral, anti-oxidant and antimicrobial $^{21,22}$. Present studies also find that polysaccharides of traditional Chinese medicine play a great role in anti-aging. For example, polysaccharides in Angelica, Lyciumbarbarum, Astragalus and so on, all exhibit distinct anti-aging action. The mechanism studies show that they work through scavenging free radicals ${ }^{23-25}$, affecting the length of telomeres at the end of chromosomes and regulating the body's immune system ${ }^{26-28}$. In this study, animal behavior experiments were used to observe the effects of AMP on the exercise spatial learning and memory abilities of D-galactose-induced aging mice, and we further explored the mechanism of AMP on NLRP3 inflammasome by AMPK/SIRT1/NF-kB signaling pathway. The 16S-RNA found that the intestinal flora of the feces of each group of mice is significantly different, which suggests that the difference in our intestinal flora also plays an important role in aging.

\section{Results}

Monosaccharide composition of AMP. As shown in Fig. 1A-C, furified polysaccharide was obtained by DEAE-52, and the monosaccharide compositionshowed that AMP was composed of Fuc (0.14\%), Rha(0.73\%), Ara (7.14\%), Gal (10.61\%), Glc (76.16\%), Xyl (2.31\%), Man (1.25\%), Gal-UA (1.43\%), Glc-UA (0.16\%), Man -UA (0.07\%). Glc was the main component, which uronic acid total ratio was 1.66 , while the peak before uronic acid was the solvent peak.

A

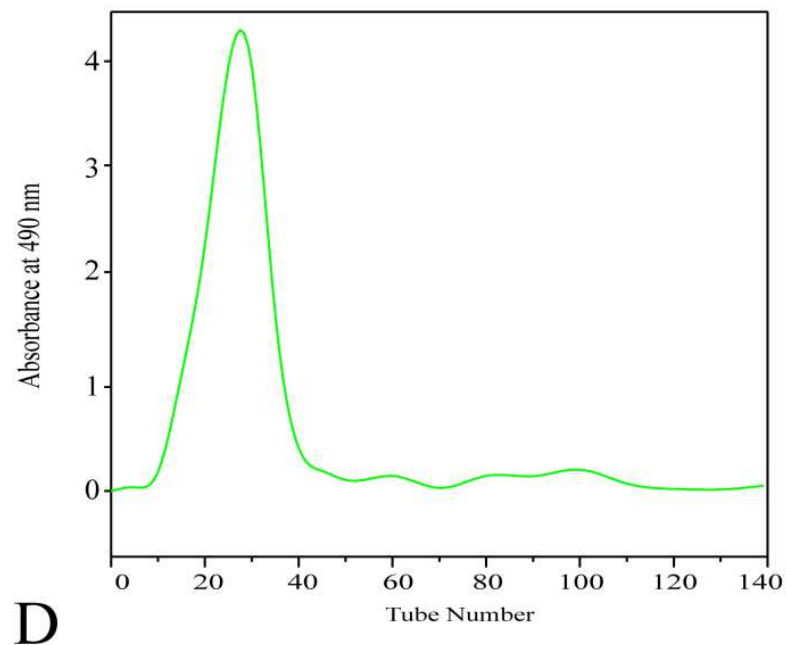

B
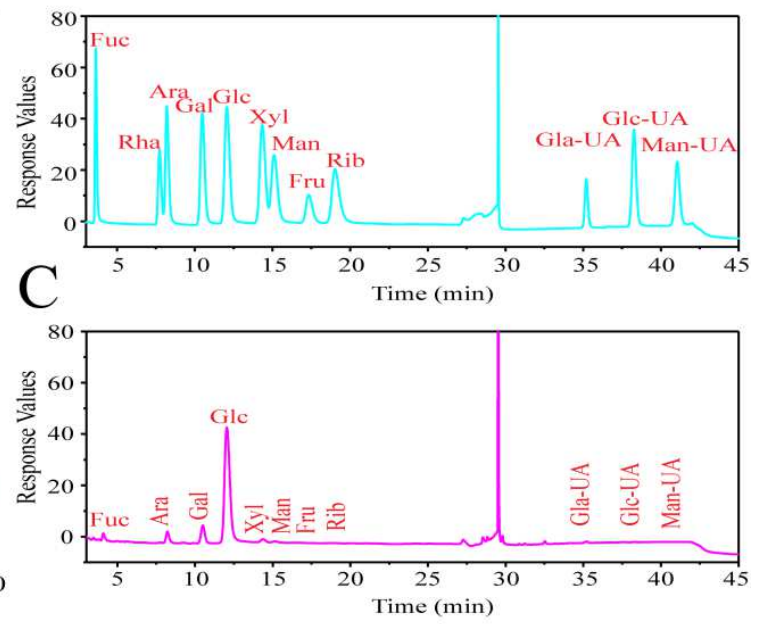

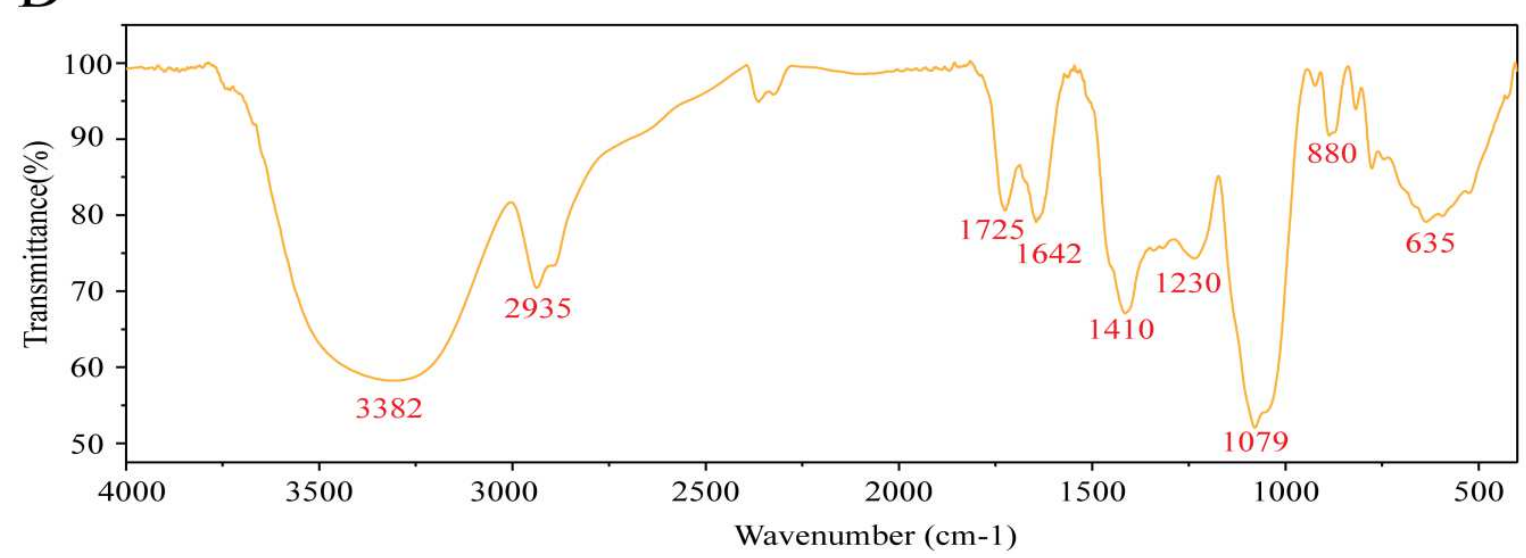


Figure 1. Analysis on the structure of AMP. (A) The purified AMP by DEAE cellulose-52. (B) The standard monosaccharides. (C) The monosaccharide composition. (D) FI-IR analysis of $A$. melanocarpa polysaccharide.

FT-IR spectra analysis of AMP. The characteristic structure of AMP was shown in Fig. 1D. 3600-3200 $\mathrm{cm}^{-1}$ was the stretching vibration absorption peak of $-\mathrm{OH}$, and the absorption peak in this region was the characteristic peak of sugars. The details were as follows: $3382 \mathrm{~cm}^{-1}$ was the $\mathrm{O}-\mathrm{H}$ stretching vibration absorption peak, which is the characteristic peak of sugars. The absorption peak at $2933 \mathrm{~cm}^{-1}$ was attributed to the C-H stretching vibration ${ }^{23}$. The absorption peak at $1650 \mathrm{~cm}^{-1}$ was attributed to $\mathrm{C}=\mathrm{O}$ stretching vibration ${ }^{25}$. The absorption peak at $1556 \mathrm{~cm}^{-1}$ was attributed to $\mathrm{C}=\mathrm{O}$ asymmetric stretching vibration $^{27}$. There was an absorption peak at $1402 \mathrm{~cm}^{-1}$, which was attributed to the C-O stretching vibration. There was an absorption peak at $1029 \mathrm{~cm}^{-1}$, which was attributed to O-H variable angle vibration. Several weak peaks at 1000-800 indicated that $\alpha$ and $\beta$ glucosides were connected to form pyranose rings ${ }^{28}$.

AMP improves the general condition of mice. The initial and final body weights of the mice were recorded and the weight gain rate was analyzed. As shown in Table 1, compared with the normal group, body weight in model group had an extremely significant effect $(p<0.01)$. AMP-H group was significantly compared with the model group $(p<0.05)$. Data showed that more than D-Gal, AMP treatment may affect the weight of mice. Moreover, the organ index of mice was recorded in Table 2, including mouse heart, liver, spleen, kidney and brain. The results showed that AMP effectively improved the organ index of mice induced by D-Gal, and the improvement effect of each organ was different.

\begin{tabular}{|c|c|c|c|c|}
\hline Group & $\begin{array}{c}\text { Dosage } \\
(\mathbf{m g} / \mathbf{k g})\end{array}$ & $\begin{array}{c}\text { Initial weight } \\
(\mathbf{g})\end{array}$ & $\begin{array}{c}\text { Final weight } \\
(\mathbf{g})\end{array}$ & $\begin{array}{c}\text { Growth rate } \\
(\mathbf{\%})\end{array}$ \\
\hline Normal & - & $33.21 \pm 1.54$ & $44.11 \pm 2.09$ & $33.09 \pm 9.17$ \\
\hline D-Gal & $200-1000$ & $33.18 \pm 0.59$ & $40.13 \pm 2.1^{* *}$ & $20.99 \pm 6.67^{* *}$ \\
\hline AMP-L & 100 & $33.28 \pm 0.70$ & $41.76 \pm 2.5$ & $25.53 \pm 7.76$ \\
\hline AMP-H & 200 & $33.18 \pm 0.59$ & $42.75 \pm 1.46^{\#}$ & $28.91 \pm 5.68^{\#}$ \\
\hline
\end{tabular}

Table 1. The effect of AMP on the body weight of aging model mice. Values represent the mean \pm S.D., $\mathrm{n}=10 .{ }^{*} p<0.05$ or ${ }^{* *} p<0.01$ vs. normal group; ${ }^{\#} p<0.05$ or ${ }^{\# \#} p<0.01$ vs. D-Gal group.

\begin{tabular}{|c|c|c|c|c|c|c|}
\hline Group & \multirow{2}{*}{$\begin{array}{c}\text { Dosage } \\
(\mathbf{m g} / \mathbf{k g})\end{array}$} & \multicolumn{4}{|c|}{ Organ index (mg/g) } \\
\cline { 3 - 7 } & - & Heart & Liver & Spleen & Kidney & Brain \\
\hline Normal & - & $0.59 \pm 0.03$ & $4.67 \pm 0.38$ & $1.7 \pm 2.19$ & $1.75 \pm 0.09$ & $1.26 \pm 0.07$ \\
\hline D-Gal & $200-1000$ & $0.43 \pm 0.03^{*}$ & $3.94 \pm 0.25^{* *}$ & $0.24 \pm 0.03^{* *}$ & $1.37 \pm 0.09^{* *}$ & $0.98 \pm 0.23^{* *}$ \\
\hline AMP-L & 100 & $0.49 \pm 0.09^{\#}$ & $3.96 \pm 0.24$ & $0.32 \pm 0.12$ & $1.49 \pm 0.18$ & $1.21 \pm 0.16^{\# \#}$ \\
\hline AMP-H & 200 & $0.52 \pm 0.08^{\# \#}$ & $4.31 \pm 0.32^{\# \#}$ & $0.53 \pm 0.63$ & $1.59 \pm 0.18^{\# \#}$ & $1.25 \pm 0.13^{\# \#}$ \\
\hline
\end{tabular}

Table 2 The effect of AMP on the organ index of aging mice. Values represent the mean \pm S.D., $\mathrm{n}=10 .{ }^{*} p<0.05$ or ${ }^{* *} p$ $<0.01$ vs. normal group; ${ }^{\#} p<0.05$ or ${ }^{\# \#} p<0.01$ vs. D-Gal group.

The mice in the normal group had smooth fur and were mentally active. The mice in the D-Gal group were curled up, their fur was sparse and there was no light, and their mental state was poor. The AMP treatment group had better fur and spirit than the D-Gal group. From the appearance of the brain of the mice, it was seen that the D-Gal group had bled spots on the surface and the distinction between the structures was blurred (Fig. 2B). The AMP treatment group seemed better than the D-Gal group. To explore the pathological conditions of the brain, we analyzed the pathological damage to the hippocampal dentate gyrus under 200 and 400 magnification eyepieces (Fig. 2C). Compared with the normal group, the granular cells in the D-Gal group are arranged irregularly, the shape of the nucleus changes, the nucleus is separated from the cytoplasm, and the cell shape is incomplete, indicating cell senescence ${ }^{26,29}$. The AMP treatment groups can significantly improve cell morphology and arrangement, especially the AMP-H dose group.

AMP improves D-Gal-induced spatial learning and memory. The hippocampus plays a key role in navigation and spatial memory ${ }^{26,30-32}$. To study the spatial memory function of mice in each group, we conducted an eight-arm maze test which could accurately and sensitively reflect the spatial learning and memory function of the brain, and make it one of the important experimental methods to detect the spatial memory of rodents ${ }^{33-35}$. The results (Fig. 3A-D) showed that the moving distance and latency of the mice in the D-Gal groups were longer, and AMP treatment could significantly improve this phenomenon, but there was no significant relationship between the latency of the mice in each quadrant. To prove this result, we performed an AchE test of mouse brain tissue (Fig. 3E). The D-Gal group was significantly higher than the normal group, and it could be reduced after AMP treatment. The above results all show that AMP can significantly improve the aging phenomenon caused by D-Gal. 

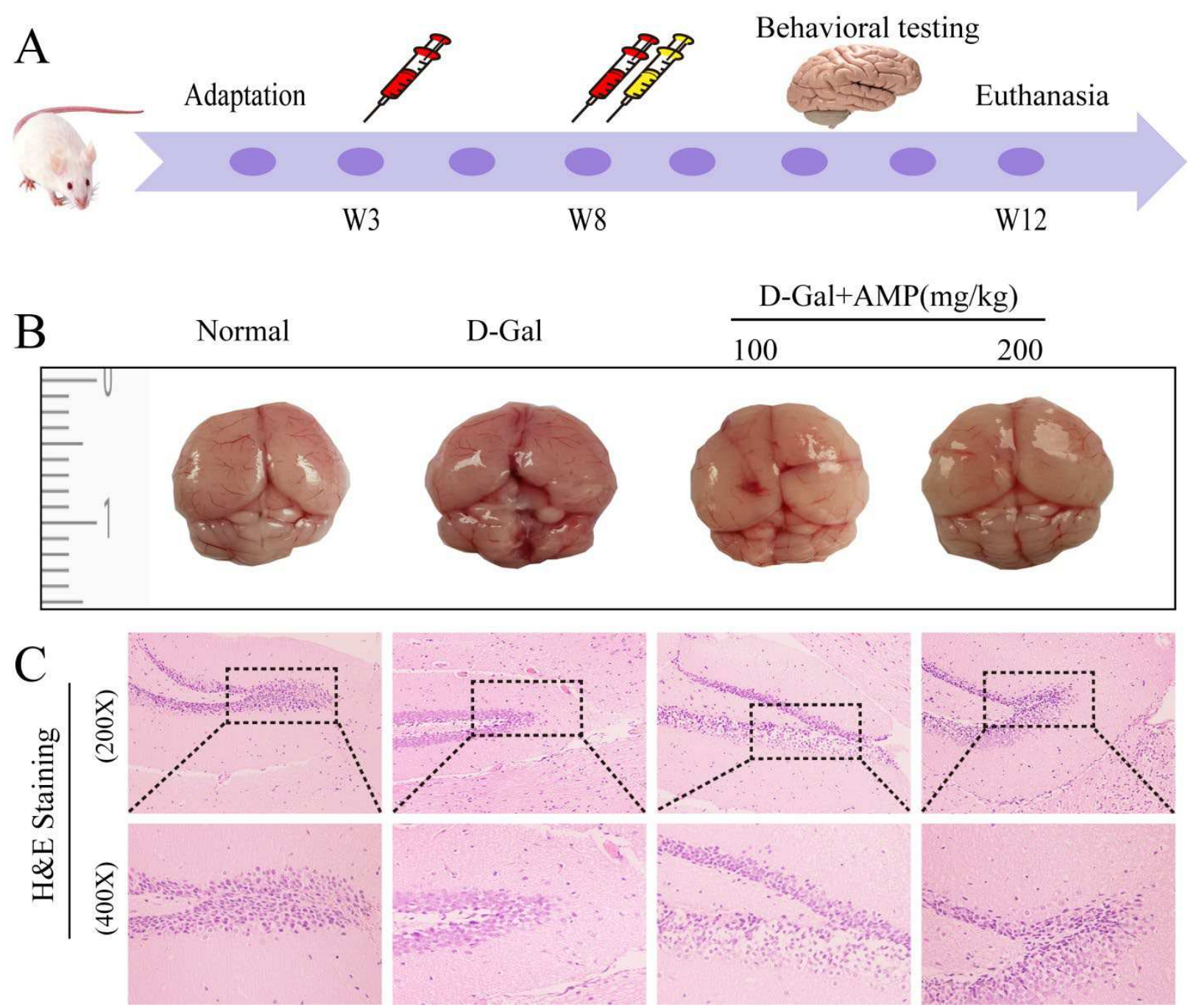

Figure 2. AMP improves the general condition of mice. (A) The experiment design of D-Gal-induced liver fibrosis model in mice. (B, C) AMP improved the appearance of the whole brain of aging mice and $\mathbf{H} \& \mathbf{E}$ staining observed the pathological damage of the hippocampus.

AMP improves D-Gal-induced oxidative stress. Because oxidative stress plays a key role in the aging process, we measured related indicators in brain tissue, such as MDA, SOD and CAT. The results are shown in Fig. 3F-H, the levels of SOD and CAT in the D-Gal group were significantly reduced, while the MDA was just the opposite. The AMP treatment group significantly improved the situation, reduced the MDA level $(p<0.01$ or $p<0.05)$, and increased the SOD and CAT levels $(p<0.01)$. These results preliminarily prove that AMP has a protective effect on oxidative stress induced by D-Gal.

To further explore the antioxidant effect of AMP on aging mice, the Nrf2/HO-1 signaling pathway was analyzed by western-blotting. The results showed that AMP significantly up-regulated the expression of Nrf2 and HO-1 nucleoprotein in DGal group $(p<0.01)$ (Fig. 3I, J), and the above results all indicated that AMP improved D-Gal-induced oxidative stress damaged. 

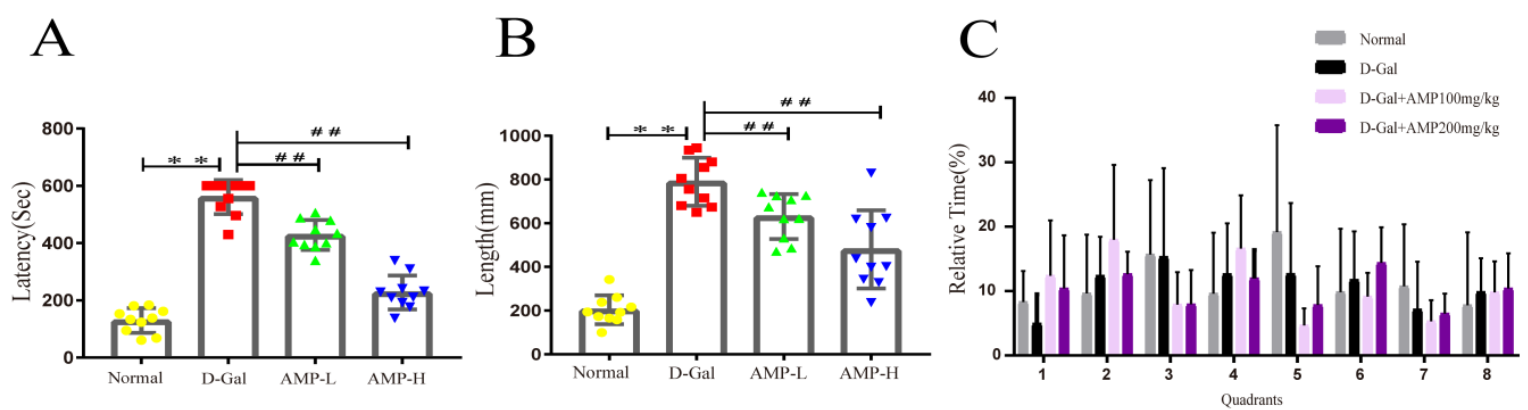

$\mathrm{D}$
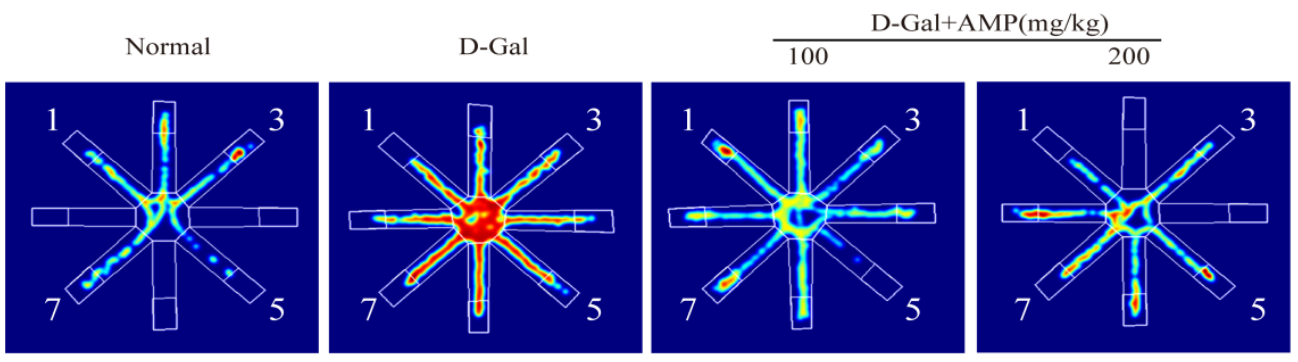

$\mathrm{E}$

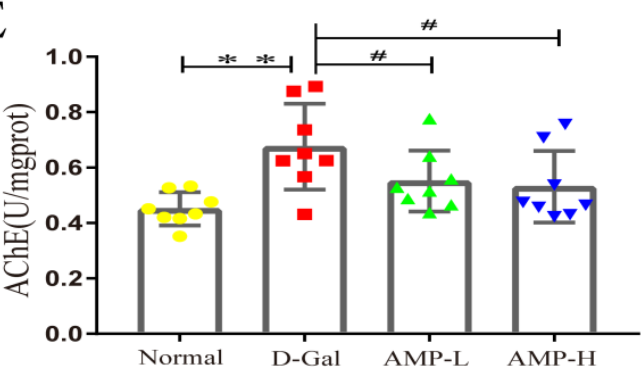

$\mathrm{G}$

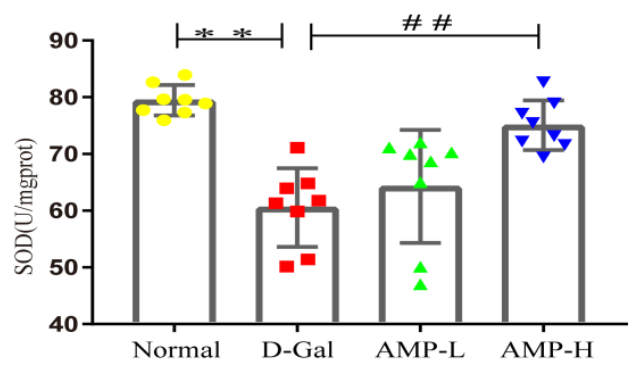

$\mathrm{F}$

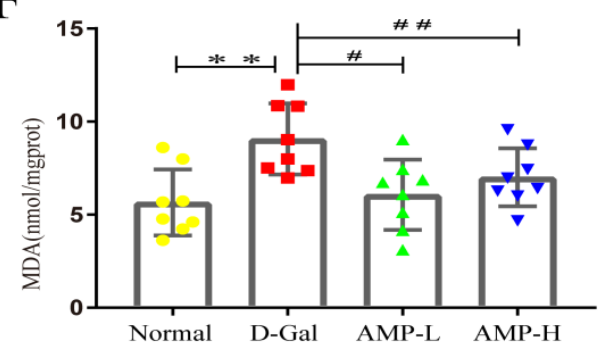

$\mathrm{H}$

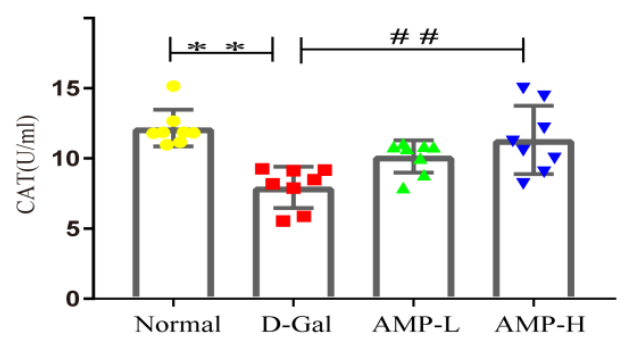

I
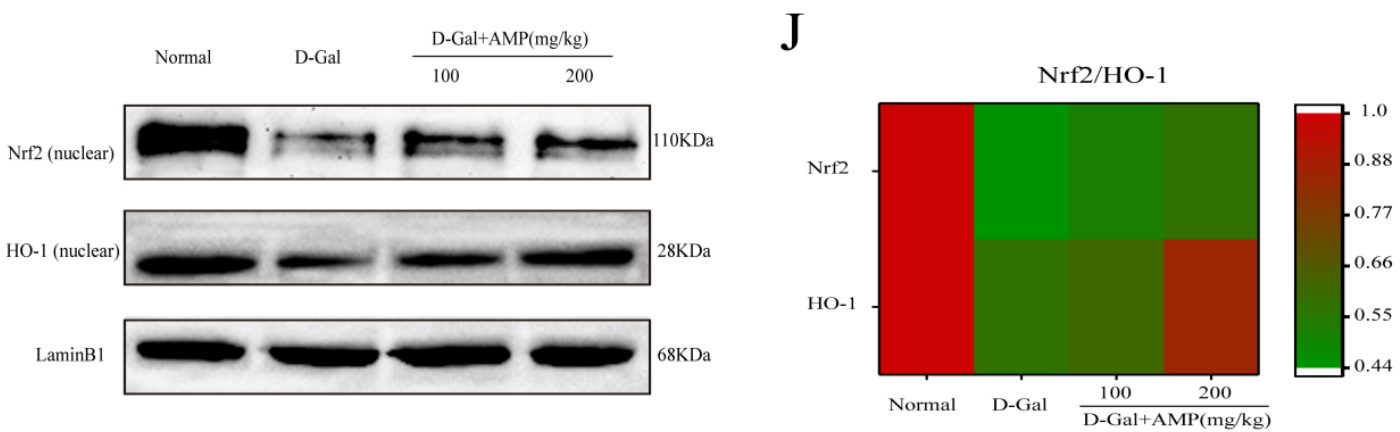

Figure 3. AMP improves D-Gal-induced spatial learning and memory. (A,B) Latent period and track length of mice in the maze. (C) Percentage of time spent by mice in each quadrant. (D) Action trajectory diagram of mice in the maze. (E) AchE level in mouse brain tissue. (F-H) The brain levels of MDA, SOD and CAT in D-Gal-induced senescence. (I, J) Nrf2 and HO-1 protein expression and heat map analysis of Nrf2 and HO-1 protein expression levels. Data are expressed as the mean \pm standard deviation (S.D), $\mathrm{n}=10 .{ }^{*} p<0.05$ or ${ }^{* *} p<0.01$ vs. normal group; ${ }^{\#} p<0.05$ or ${ }^{\# \#} p<0.01$ vs. D-Gal group. 
AMP inhibits the production of NALRP3 inflammasome through AMPK. The circulatory regulation mechanism between AMPK and SIRT1 reinforces the important role of energy metabolism balance in the aging process. SIRT1 participates in the regulation of aging process by reducing the activity of the $\mathrm{p} 53$ gene $^{14,15}$. Therefore, we tested the aging-related proteins, and the results are shown in Fig. 4A-D. The expression of AMPK and SRIT1 in the model group was significantly less, while P53 was the opposite.

To explore whether AMP has an anti-pyroptotic effect on D-Gal-induced senescence, we determined by detecting the levels of related proteins in the classical pyroptotic pathway mediated by Caspase-1. The results showed that in Fig. 4E-H, the protein expressions of GSDMD, Caspase-1, and IL-1 $\beta$ in the D-Gal group were all increased, and the AMP treatment group could change this phenomenon. The occurrence of pyrolysis is often accompanied by the accumulation of inflammasomes. In order to further explore the molecular mechanism of AMP against pyrolysis, we focused on the expression of NLRP3 and ASC proteins. The results showed the compared with the normal group, the protein content of NLRP3 and ASC in the D-Gal group increased, and their expression decreased after AMP treatment (Fig. 4I-K). All the above results showed that AMP can alleviate the pyrolysis by D-Gal-induced, thereby delaying the aging state of mice.
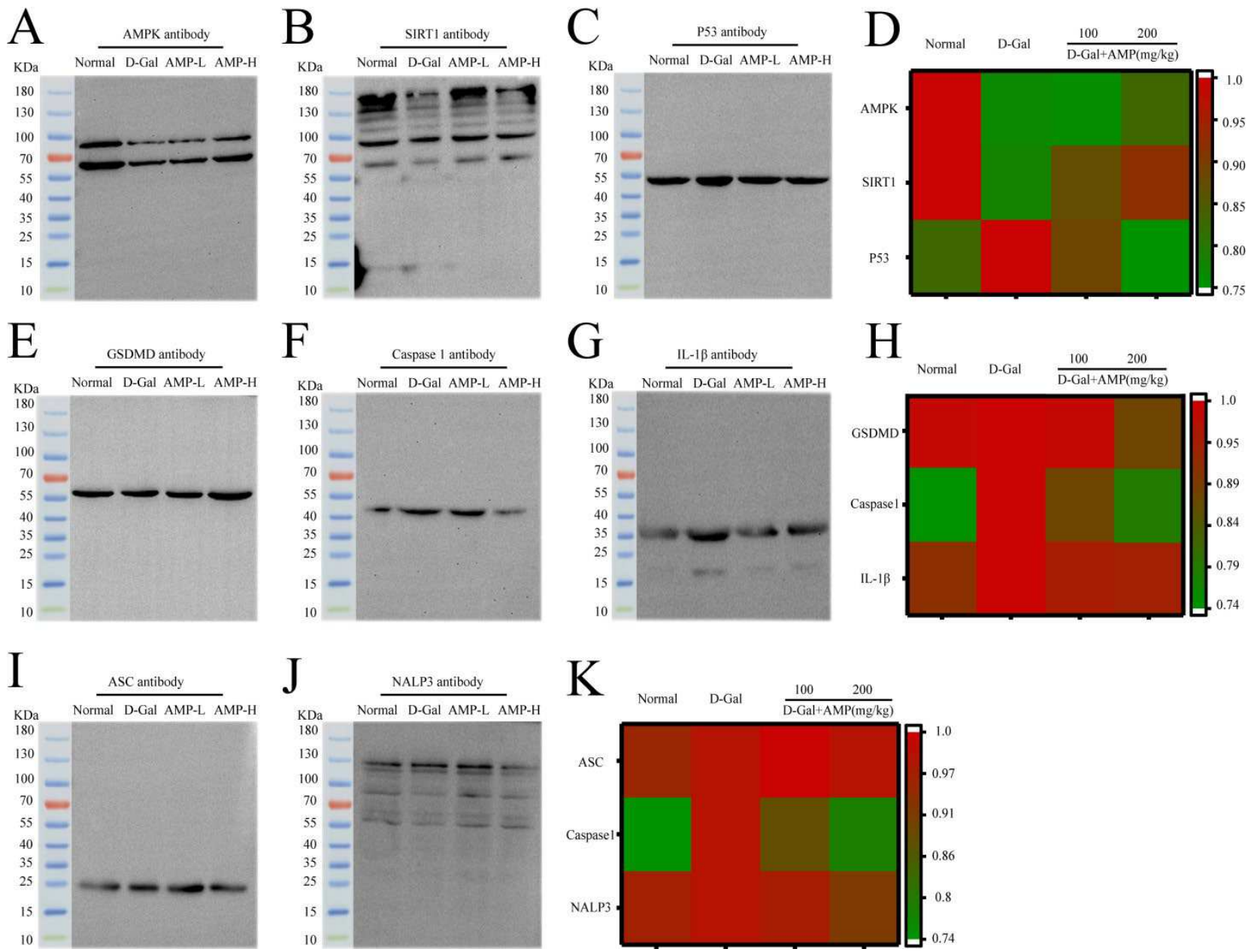

Figure 4. AMP improves D-Gal-induced oxidative stress. (A-D) AMPK, SIRT1 and P53 proteins expression, and heat map analysis of AMPK signaling pathway expression levels. (E-K) The inflammasome proteins expression of GSDMD, Caspase 1, IL-1 $\beta$, ASC and NALP3, and heat map analysis of inflammasome protein expression levels. Data are expressed as the mean \pm standard deviation (S.D), $\mathrm{n}=10 .{ }^{*} p<0.05$ or ${ }^{* *} p<0.01$ vs. normal group; ${ }^{\#} p<0.05$ or ${ }^{\# \#} p<0.01$ vs. D-Gal group.

AMP reduces D-Gal-induced inflammation. To explore whether AMP had an anti-inflammatory effect on D-Gal-induced senescence, the level of related proteins were determined in the NF- $\mathrm{KB}$ classic inflammatory pathway. The use of D-Gal caused

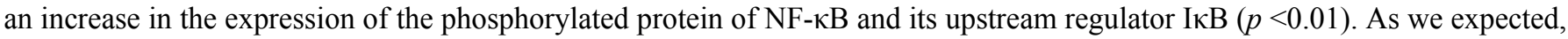
the AMP treatment group could significantly inhibit the high expression of NF- $\mathrm{KB}$ and block the increase in the expression of the upstream regulatory factor IאB- $\alpha$ phosphorylated protein $(p<0.01)$ (Fig. 5A, B). All the above results indicated that AMP can improve D-Gal-induced aging symptoms through anti-inflammatory effects. 

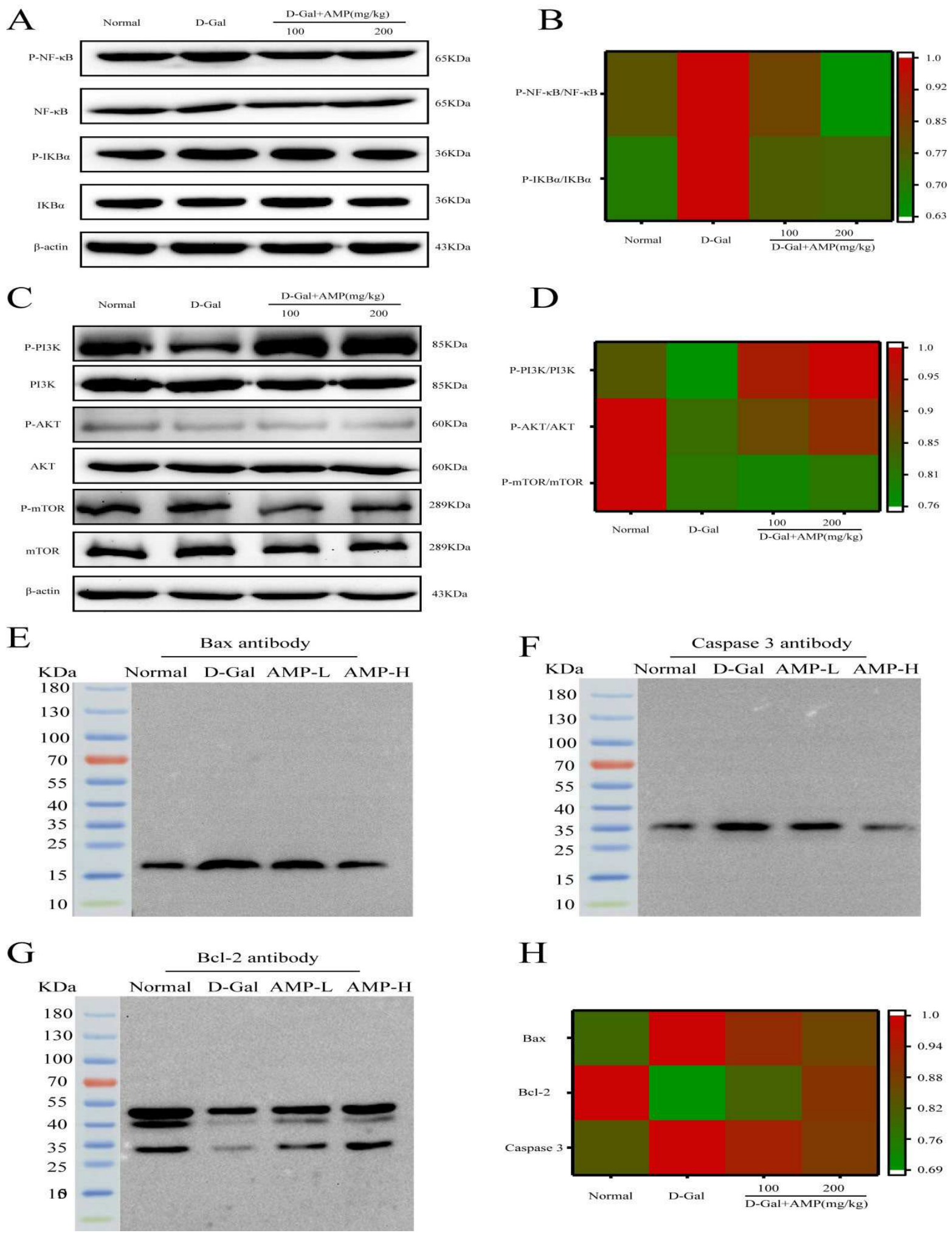

Figure 5. AMP exerts the anti-aging effect in mice by regulating NF- $\mathrm{kB}-$ mediated inflammation and PI3K/AKT/mTOR anti-

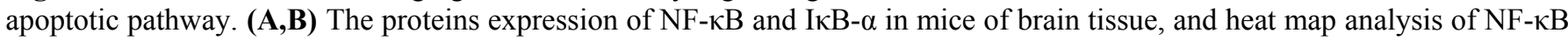
signaling pathway expression protein levels. (C-H) The proteins expression of p-PI3K/PI3K, p-AKT/AKT, p-mTOR/mTOR, Bax, Caspase 3 and Bcl-2 in mice of brain tissue, and heat map analysis of PI3K/AKT/mTOR and its downstream proteins levels. Data are expressed as the mean \pm standard deviation (S.D), $\mathrm{n}=10 .{ }^{*} p<0.05$ or ${ }^{* *} p<0.01$ vs. normal group; ${ }^{\#} p<0.05$ or ${ }^{\# \#} p$ $<0.01$ vs. D-Gal group. 
AMP reduces D-Gal-induced apoptosis through PI3K/AKT pathway. We performed PI3K/AKT-related proteins including downstream apoptotic proteins Bax, Bcl-2, and Caspase3. As shown in Fig. 5C-H, the AMP treatment group significantly increased the expression of P-PI3K, P-AKT, and P-mTOR proteins compared with the D-Gal group $(p<0.05$ or $p<0.01)$. Furthermore, for its downstream apoptotic proteins, AMP significantly reduced the expression of Bax and Caspase 3 proteins, while the results of Bcl-2 were just the opposite $(p<0.05$ or $p<0.01)$. These results indicated that AMP inhibited apoptosis by D-Gal-induced.

AMP improves D-Gal-induced aging by regulating the intestinal flora of mice. The results of the Venn diagram showed that the number of OTUs in the normal group, D-Gal group, AMP-L, and AMP-H groups are 473,520,509,467, respectively, there were 435 OTUs in the normal group and the D-Gal group. D-Gal group and AMP-L, AMP-H group had a total of 458,434 OTUs; AMP-L and AMP-H group have a total of 429 OTUs (Fig. 6A). According to the species abundance table, the relative abundance of the Bacteroides and Firmicutes phylums were relatively increase in the four groups. After AMP treatment, the relative abundance of the Bacteroides phylum increases, and the relative abundance of Firmicutes decrease (Fig. 6B). The dominant bacterial groups that were significantly enriched in the four groups were: lactobacillus, akkermansia, bacteroides, prevotella, barneslella, and allstipes, but there was no significant difference between the groups (Fig. 6F).
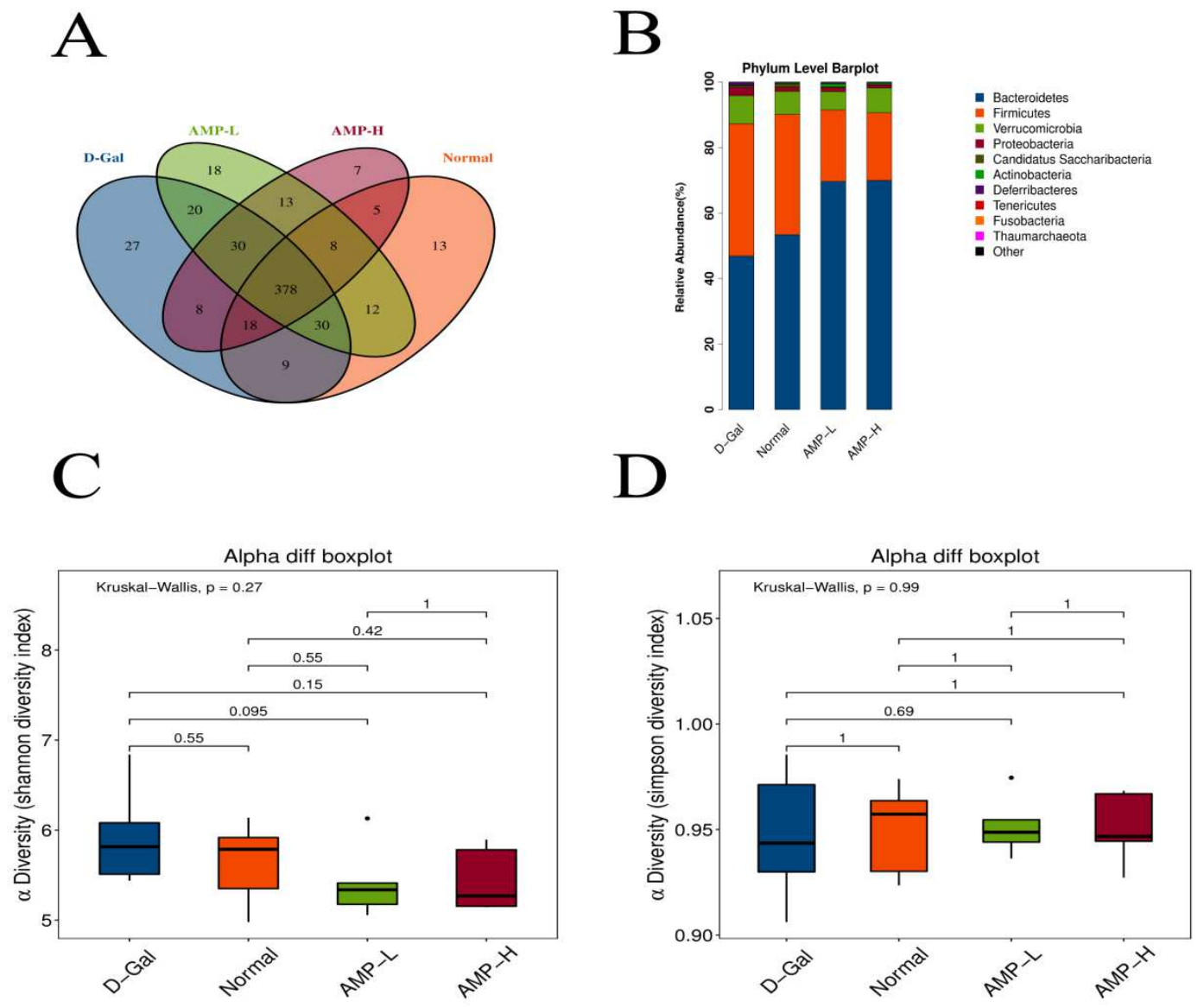

Figure 6. Continued.

As shown in Fig. 6C and D, $\alpha$ diversity (Shannon and Simpson index) increased from $0.27-0.99$. The $\beta$ diversity analysis based on Weighted Unifrac clustering (pCoA, Anosim) showed that the difference between the four groups of mice was significantly greater than the difference within the group, and there are significant differences $(\mathrm{R}=0.367, \mathrm{P}=0.001)$ (Fig. 6E). The pCoA diagram showed that although the flora in the four groups had indeed changed from varying degrees, the flora of the AMP-L group was basically the same as the normal group of mice, while the flora of the AMP-H group was significantly different from the rest (Fig. 6G). 


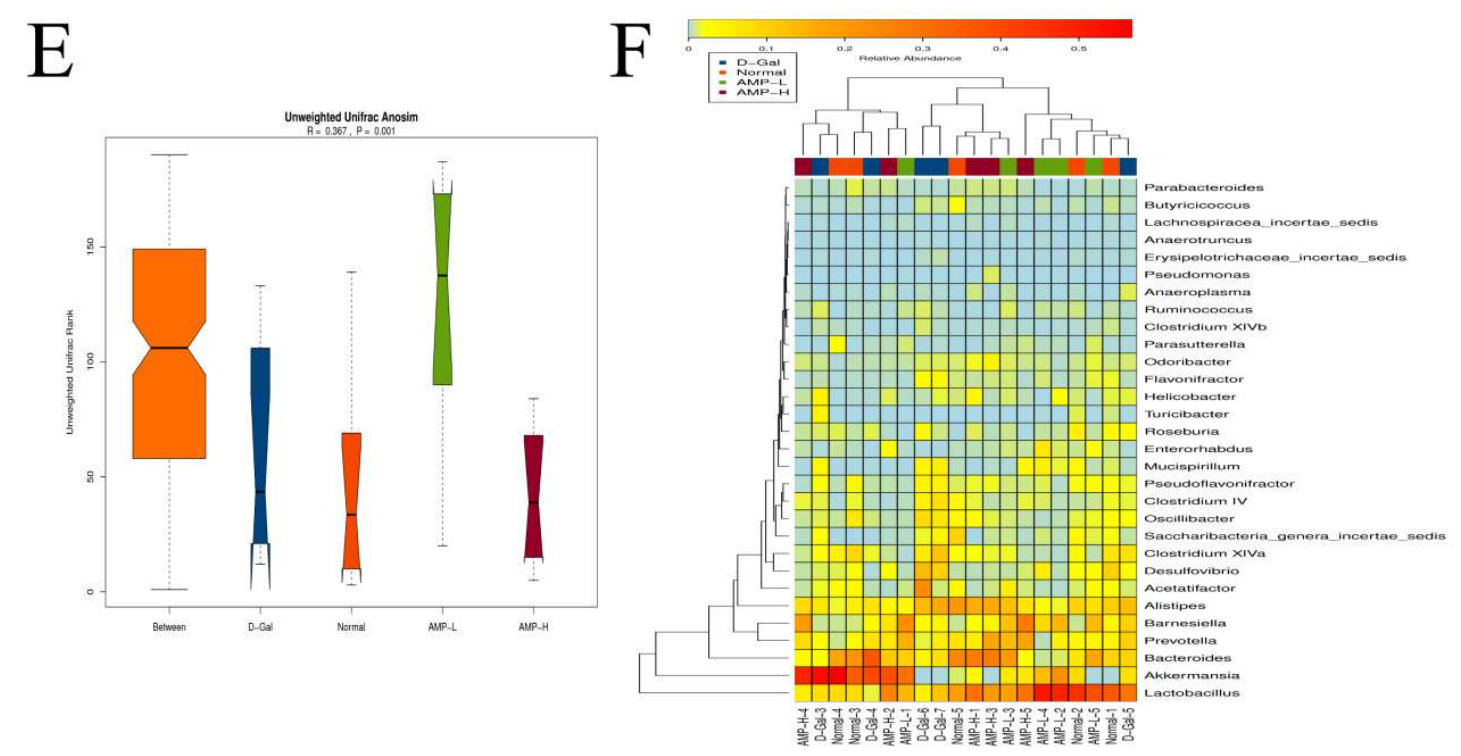

$\mathrm{G}$
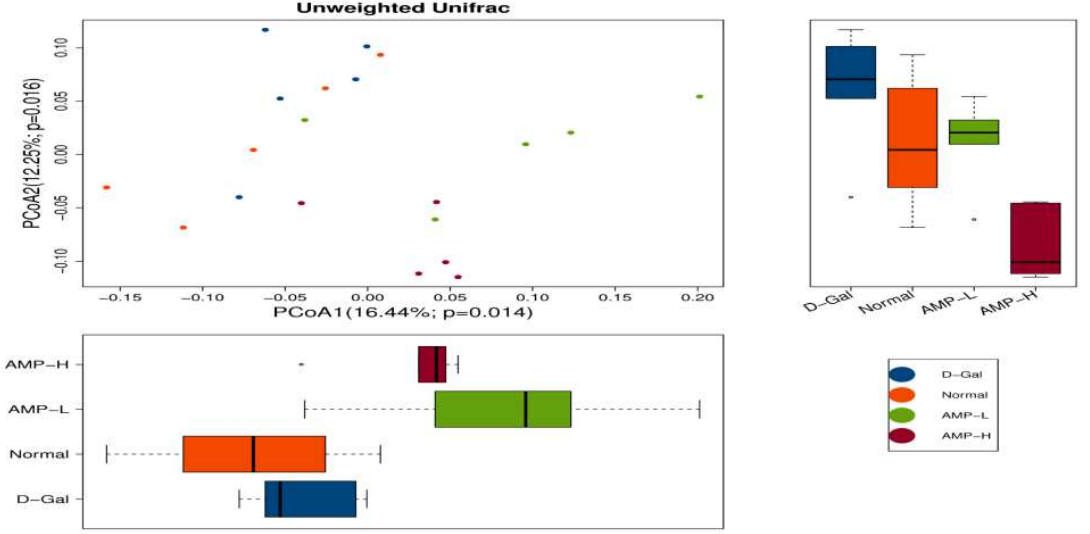

Figure 6. AMP improves D-Gal-induced aging by regulating the intestinal flora of mice. (A) Venn diagram; (B) Phylum lever Barplot. (C,D) Alpha diff boxplot. (E) Unweight UnifracAnosim. (F,G) Relative Abundance and unweighted Unifrac. Data are expressed as the mean \pm standard deviation (S.D), $\mathrm{n}=10 .{ }^{*} p<0.05$ or ${ }^{* *} p<0.01$ vs. normal group; ${ }^{*} p<0.05$ or ${ }^{\# \#} p<0.01$ vs. DGal group.

\section{Discussion}

D-galactose, as a reducing sugar, can induce oxidative stress and tissue damage, lead to cell apoptosis, mitochondrial dysfunction, and decrease antioxidant defense system function ${ }^{30-32}$. It was also used on rodents to get a long-term preclinical agingmodel ${ }^{36,37}$. Lots of research showed that medicinal polysaccharides could effectively prevent senescence ${ }^{38,39}$. While, Aronia melanocarpa polysaccharides as its main component were only reported for its anti-hypertensive and anti-atherosclerotic activity. To evaluate the anti-aging effect of AMP by measuring the body weight, organ index, levels of MDA, SOD, and CAT, whichwere widely used as indicators of aging.Compared with D-gal group, AMP treatment could significantly increase the weight growth of aging mice, and their heart, liver, kidney, brain all exhibited different degrees improvement. In addition, eight-arm maze test was a common behavioral tested used to evaluate spatial work and reference memory in mice. The results showed that AMP could significantly reduce the aging mice the feeding distance and time of aging mice. The outwards appearance observation included fur, mental state and activity also proved the protective effect of AMP on aging. H\&E analysis showed that amp treatment alleviated D-gal-induced brain injury. Interestingly, the results of AchE were the same as we expected, AMP can improve neurological function

As well-known, the intestinal microbes are called the "second genome", which could affect the body's state during aging, including microbes, genomic DNA, proteins and metabolites ${ }^{40-42}$. In aging mice, the diversity of intestinal biota decreased, with beneficial bacteria decreasing and facultative anaerobes increasing, which caused cognitive and memory functions of the elderly decline ${ }^{36,37,43,44}$. In order to figure out whether AMP can alleviate the aging state of the body by improving the mouse 
flora, collected mouse feces for 16sRNA analysis. The results showed that the proportion of the beneficial bacteria Bacteroides phylum was significantly increased, the $\beta$ diversity analysis based on weighted Unifrac clustering (pCoA, Anosim) showed that the difference between the four groups $(\mathrm{R}=0.367, \mathrm{P}=0.001)$ was significantly greater than the difference within groups. In summary, AMP had obvious impacts on intestinal flora, which opening up a new path of aging-related research.

About the mechanism study, we considered the oxidative stress, inflammation and apoptosis aspects which were proved to be associated with aging. As a key factor of the regulation of bioenergy metabolism, AMPK plays a vital role in antiinflammatory and growth regulation, which is also the core of metabolic-related diseases ${ }^{45,46}$, it is related to multiple signaling pathways such as SIRT1, NF-KB, mTOR, P53, etc. P53 tumor suppressor protein is a multifunctional transcription factor that regulates cell proliferation, cycle and apoptosis. When cells are damaged and become cancerous, p53 will induce cell aging and prevent them from further malignant transformation into tumor cells. This fact shows that the level of P53 will increase to age ${ }^{47}$. Our results also confirmed this phenomenon.

As a pivot protein, we focused on the role of the AMPK/SIRT1/NF-KB signaling pathway to aging and the chronic inflammation associated with aging. The increased trans-regulatory activity of NF-kB complex about age and the association with chronic inflammation with increased NF- $\mathrm{kB}$ activity and metabolic diseases such as diabetes and atherosclerosis suggest the role of NF- $\mathrm{KB}$ signaling in the aging process ${ }^{48}$. Metformin is known to be in clinical trials in Europe as an anti-aging agent, an agonist for AMPK, which also inhibits the NF- $\mathrm{KB}$ signaling pathway for anti-inflammatory effects ${ }^{49}$. NF- $\mathrm{kB}$ promotes the transcription and translation of the NLRP3 inflammasome molecules, and then activates NLRP3 inflammasome in response to inflammasome activators, releasing inflammatory factors ${ }^{50}$. Combines with ASC and promotes the maturation of IL-1 $\beta$ inflammatory factors, and induces cell pyroptosis. Inflammatory bodies are accompanied by pyroptosis, for this reason, we also measured the expression levels of Caspase1 and GSDMD, GSDMD also has been called the "executioner" of pyroptosis ${ }^{51}$. These findings suggest that an indirect regulatory effect of AMPK on NF- $\mathrm{KB}$, which may explain the decreased activity of AMPK with aging, resulting in energy metabolism imbalance and inflammatory response.

There is no doubt that oxidative stress is a key factor of the pathogenesis of neurodegenerative diseases ${ }^{52}$. Nrf2 is a key transcription factor regulating oxidative stress and exists on the cytoplasm under normal physiological conditions. Once activated, it enters the nucleus and regulates the expression of downstream proteins and plays an anti-inflammatory effect ${ }^{53}$. Heme oxygenase-1 (HO-1) is one of the most widely distributed antioxidant enzymes in the body, with anti-inflammatory effects $^{54,55}$. In our research, we found that AMP treatment can promote the entry to Nrf2 Nuclear and up-regulate the expression of HO-1 and inhibit the protein expression. Tested the indicators related to oxidation MDA, SOD, CAT, these results are the same as we expected. Studies have shown that the PI3K/Akt/mTOR pathway is one of the most important upstream signals for regulating Nrf2 nuclear translocation and the expression of second-stage antioxidant enzymes ${ }^{56-58}$. We explored the $\mathrm{PI} 3 \mathrm{~K} / \mathrm{Akt} / \mathrm{mTOR}$ pathway-related proteins and tested the downstream apoptotic proteins. Family proteins including Bax and Bcl-2 are typical proteins that regulate cell proliferation and apoptosis ${ }^{59}$. In this study, AMP increased the expression of P-PI3K and P-AKT, successfully inhibited the expression of Bax and Caspase 3 and enhanced the expression of Bcl-2 through the PI3K/AKT pathway. These findings indicated that AMP had an anti-oxidative stress effect, passed through the $\mathrm{PI} 3 \mathrm{~K} / \mathrm{AKT} / \mathrm{mTOR}$ signaling pathway, and can play an anti-apoptotic effect.

\section{Conclusions}

In conclusion, AMP was confirmed to be active in anti-aging by the basic characteristics and indicators of mice, behavioral space, learning, memory functions, and intestinal flora analysis. Then we comprehensively analyzed its possible mechanism on the oxidative stress, inflammation, apoptosis and other aspects. It was found that AMP inhibits NLRP3 inflammasome by AMPK/SIRT1/NF- $\kappa \mathrm{B}$ signaling pathway and regulate intestinal flora delays aging in mice, and these findings describe the potential mechanism of AMP for the prevention of aging. However, aging is actually a comprehensive manifestation of the whole body, this study only detected the brain, and the impact on AMP on other organs remains further studies.

\section{Materials and Methods}

Chemicals and materials. Aspartate aminotransferase (AST), alanine aminotransferase (ALT), reduced glutathione (GSH), superoxide dismutase (SOD), malondialdehyde (MDA) threonine from Nanjing Jiancheng Bioengineering Institute LigninEosin (H\&E) commercial assay kits. D-gal (purity $\geq 95 \%$ ) was purchased from Sigma (USA). Western Blot related antibodies were purchased from Proteintech and Arigo (Changchun, China).All other chemicals and reagents, used in the study, were of analytical grade.

Preparation of AMP. Separation and Furification of Aronia polysaccharides. Wash the fresh fruits, extract the polysaccharides by decoction in distilled water, concentrate the decoction to one-eighth of the original volume, and then add 
three times the volume of $95 \%$ ethanol to precipitate overnight in a refrigerator at 4 degrees to obtain a precipitate. Add water to dissolve and continue. Alcohol precipitation was repeated three times to obtain the crude polysaccharides after defatting, depigmentation, and preliminary purification by macroporous resin D101, vacuum freeze-drying to constant weight, and recording as AMP. After preliminary purification by the macroporous resin, one gram of AMP was dissolved in deionized water. It uses deionized water first, and then uses $0.1,0.3$ and $0.5 \mathrm{~mol} / \mathrm{L} \mathrm{NaCl}$ solution (flow rate of $1.0 \mathrm{~mL} / \mathrm{min}$ ) to elute in DEAE-52 fiber column $(3 \times 40 \mathrm{~cm})$ for classification. The diameter of DEAE-52 cellulose is 300 meshes. The eluate is collected by a fully automatic collector, each tested tube $(10 \mathrm{~mL})$ is collected every $10 \mathrm{~min}$, for a total of 150 tubes. The polysaccharide in the eluate was detected by the sulfuric acid phenol method. Measure the absorbance value and draw an elution curve to determine the composition of the MWP. After vacuum freeze-drying, a fure light brown-yellow powdered was obtained, which was used for subsequent structure identification.

Fourier-transformed infrared (FT-IR) spectroscopic analysis. The sample was weighed $2 \mathrm{mg}$ and $200 \mathrm{mg}$ of potassium bromide and pressed into tablets, while the blank control were pressed using potassium bromide powder. The samples were scanned and recorded on a Fourier transform infrared spectrometer FT-IR650 (Tianjin Gangdong Science and Technology Development Co., Ltd.).

Monosaccharide composition analysis. The monosaccharide composition of AMP was determined by ion chromatography. Heat $5 \mathrm{mg}$ of sample and TFA at $121^{\circ} \mathrm{C}$ for $2 \mathrm{~h}$, dry with nitrogen, remove residual TFA with methanol and repeat three times.

The chromatographic system adopts the Thermo ICS5000 $0^{+}$ion chromatography system (Thermo Fisher Scientific, USA), Dionex $^{\mathrm{TM}}$ CarboPac ${ }^{\mathrm{TM}}$ PA10 $(250 \times 4.0 \mathrm{~mm}, 10 \mu \mathrm{m})$ liquid chromatography column, the injection volume is $20 \mu \mathrm{L}$, column temperature is $30^{\circ} \mathrm{C}$. M obile phase $\mathrm{A}\left(\mathrm{H}_{2} \mathrm{O}\right)$ and mobile phased $\mathrm{B}(100 \mathrm{mM} \mathrm{NaOH})$.

Fucose (Fuc), rhamnose (Rha), arabinose (Ara), galactose (Gal), Glucose (Glc), xylose (Xyl), mannose (Man), Galacturonic acid (Gal-UA), Glucuronic acid (Glc-UA), Mannuronic acid (Man-UA) are used as standard monosaccharides. The method of their treatment is the same as that of sample analysis, and qualitative and quantitative are performed according to the retention time of chromatographic peak.

Analysis of the anti-aging activity of polysaccharide. Animals and experimental design. 40 adult male ICR mice (6-8 weeks old, weight 22-25 g) were purchased from Changchun Yisi Experimental Animal Co., Ltd., with quality certificate SCXK (JI) 2019-0008 (Changchun, China). Mice were maintained under constant temperature and humidity in pathogen free conditions at a 12:12 h (L:D) photoperiod, and unlimited access to food and water. All animal investigational processes were done according to the Guide for the Attention and usage of Laboratory Animals and permitted by the Animal Investigational Morals Committee of Jilin Agricultural University, ethics approval number: 2019-08-28-001.

As shown in Fig. 2A, we have established the basic flow of experimental operations. After 3 weeks of adaptation, the mice were randomly divided into four groups: normal group, D-Gal group, AMP low-dose group and high-dose group, with 10 mice in each group. In the D-Gal group, the dose was increased by $200 \mathrm{mg} / \mathrm{kg}$ by intraperitoneal injection until the dose was increased to $1000 \mathrm{mg} / \mathrm{kg}$ to maintain the same dose, once per 3 days for 12 weeks, and administer AMP (100 and $200 \mathrm{mg} / \mathrm{kg}$ ) were given daily by oral gavage after 6 weeks of D-Gal-induced. The same volume of saline weregiven to the normal group. Twenty-four hours after the last dose, all mice were executed and dissected to get the blood supernatant, which was isolated after centrifugation at $4^{\circ} \mathrm{C}$ for $10 \mathrm{~min}$ and stored at $-80^{\circ} \mathrm{C}$ until analysis. Harvest brain, heart, kidney, spleen and liver tissues to calculate the organ index: organ weight index $=$ organ weight $(\mathrm{mg}) /$ body weight $(\mathrm{kg})$. Use physiological saline in a ratio of 1:9 and grind it with a tissue grinder at $4^{\circ} \mathrm{C}$. The separated homogenate supernatant was centrifuged at $4000 \times \mathrm{g}$ at $4{ }^{\circ} \mathrm{C}$ for $10 \mathrm{~min}$, and then stored at $-80^{\circ} \mathrm{C}$ to measure cholinergic function. Brain tissue samples were then fixed in formalin for histological examination, and other sections were rapidly stored frozen in liquid nitrogen.

Tissue collection and sample preparation. Record the initial and final weight of mice were collected the feces of mice and immediately placed in liquid nitrogen to be frozen for subsequent intestinal flora analysis. After fasting for $12 \mathrm{~h}$, blood was collected by orbital blood collection, and determination of basic oxidation indexes. The brain was immediately removed and stored at $-80^{\circ} \mathrm{C}$ for biochemical analysis. Mouse organs were collected and weighed for organ index analysis.

Biochemical analysis. Fresh blood was centrifuged at $3000 \mathrm{rpm}, 4^{\circ} \mathrm{C}$ for $30 \mathrm{~min}$, serum was collected, and the contents of glutathione (GSH), superoxide dismutase (SOD), and malondialdehyde (MDA) were used to evaluate oxidation. The brains of mice were homogenized with $0.9 \%$ saline, and the acetylcholinesterase ( $\mathrm{AChE}$ ) content was determined to evaluate the damage of neurons in the brain.

Histological analysis. After the mice were dissected, fresh brains of the mice were obtained, embedded in paraffin, and were 
detected using a hematoxylin and eosin (H\&E) staining kit according to the manufacturer's instructions. The results were obtained through optical microscope (Leica DM4B, Germany).

Western blot. A protein extraction kit (Thermo) was used to obtain brain supernatant. The samples were then separated on SDS polyacrylamide gel and transferred to PVDF membrane, blocked with BSA for $1.5 \mathrm{~h}$, washed with TBST for 5 min three times, and incubated with different antibodies overnight. The membrane was rinsed three times in TBST for 5 min each time, and then incubated with the HRP-labeled antibody for $1 \mathrm{~h}$. ECL reagent (General Electric Medical Group, USA) was used in the membrane, and the specific protein was detected by the Fluorchem device.

Behavioral testing. Eight-arm maze was used to test the space and learning ability of mice. Before testing, we comforted the mice at a fixed time to adapt to the environment, and they were fasted for $12 \mathrm{~h}$ then tested for $10 \mathrm{~min}$, four arms were randomly selected for sugar pill induction. Use RMT-100 analysis software (Chengdu Taimeng Software Co., Ltd.) to collect data and its motion trajectory parameters were recorded. Analyzing the distance moved by the mouse during the experiment, the incubation period, and calculate the time/total time for the mouse to pass each quadrant.

16S rDNA high-throughput sequencing. A total of twenty stool samples were randomly selected from all groups for intestinal flora analysis (five samples per group). High-throughput sequence of the 16S V4 region was used to analyze and compare the intestinal flora. Using Uparse software, based on the OTU threshold of $97 \%$ similarity, the sequences were clustered into operational taxonomies (OTU). Based on the sequence reads and OTU, the $\alpha$ diversity, $\beta$ diversity and linear discriminant analysis effect size (LEfSe) were analyzed.

Statistical analysis. Statistical analysis was carried out using SPSS statistical software. The numerical comparison was performed by ANOVA test for analyzing the differences between the two groups. The results are shown as the mean $\pm \mathrm{SD}$, and the significance level is defined as $p<0.05$.

\section{References}

1. Ding, Q. et al. Antioxidant and anti-aging activities of the polysaccharide TLH-3 from Tricholoma lobayense. Int. J. Biol. Macromol.85, 133-140 (2016).

2. Lin, L. et al. Antioxidative and renoprotective effects of residue polysaccharides from Flammulina velutipes. Carbohydr. Polym.146, 388-395 (2016).

3. Govindan, S. et al. Antioxidant and anti-aging activities of polysaccharides from Calocybe indica var. APK2. Exp. Toxicol. Pathol.68, 329-334 (2016).

4. Baar, M. P. et al. Targeted Apoptosis of Senescent Cells Restores Tissue Homeostasis in Response to Chemotoxicity and Aging. Cell169, 132-147.e16 (2017).

5. Shay, J. W. Role of telomeres and telomerase in aging and cancer. Cancer Discovery vol. 6 584-593 (2016).

6. A, L.-P. et al. Corrigendum: Mitochondrial and nuclear DNA matching shapes metabolism and healthy ageing (Nature (2016) 535 (561-565) DOI: 10.1038/nature18618). Nature vol. 542124 (2017).

7. Wang, Y. \& Hekimi, S. Mitochondrial dysfunction and longevity in animals: Untangling the knot. Science vol. 350 1204-1207 (2015).

8. Sun, N., Youle, R. J. \& Finkel, T. The Mitochondrial Basis of Aging. Molecular Cell vol. 61 654-666 (2016).

9. Kaushik, S. \& Cuervo, A. M. Proteostasis and aging. Nature Medicine vol. 21 1406-1415 (2015).

10. Reznick, R. M. et al. Aging-Associated Reductions in AMP-Activated Protein Kinase Activity and Mitochondrial Biogenesis. Cell Metab.5, 151-156 (2007).

11. Fryer, L. G. D. \& Carling, D. AMP-activated protein kinase and the metabolic syndrome. in Biochemical Society Transactions vol. 33 362-366 (Biochem Soc Trans, 2005).

12. Qiang, W., Weiqiang, K., Qing, Z., Pengju, Z. \& Yi, L. Aging impairs insulin-stimulated glucose uptake in rat skeletal muscle via suppressing AMPK $\alpha$. Exp. Mol. Med.39, 535-543 (2007).

13. Borodkina, A. V. et al. Tetraploidization or autophagy: The ultimate fate of senescent human endometrial stem cells under ATM or p53 inhibition. Cell Cycle15, 117-127 (2016).

14. Giannakou, M. E. \& Partridge, L. The interaction between FOXO and SIRT1: Tipping the balance towards survival. Trends in Cell Biology vol. 14 408-412 (2004).

15. Galluzzi, L., Kepp, O. \& Kroemer, G. TP53 and MTOR crosstalk to regulate cellular senescence. Aging vol. 2 535-537 (2010).

16. Chrubasik, C., Li, G. \& Chrubasik, S. The clinical effectiveness of chokeberry: A systematic review. Phytotherapy Research vol. 24 1107-1114 (2010).

17. Hukkanen, A. T., Pölönen, S. S., Kärenlampi, S. O. \& Kokko, H. I. Antioxidant capacity and phenolic content of sweet rowanberries. J. Agric. Food Chem.54, 112-119 (2006). 
18. Kokotkiewicz, A., Jaremicz, Z. \& Luczkiewicz, M. Aronia plants: a review of traditional use, biological activities, and perspectives for modern medicine. Journal of medicinal food vol. 13 255-269 (2010).

19. Tian, Y. et al. Phenolic compounds extracted by acidic aqueous ethanol from berries and leaves of different berry plants. Food Chem.220, 266-281 (2017).

20. Vagiri, M. \& Jensen, M. Influence of juice processing factors on quality of black chokeberry pomace as a future resource for colour extraction. Food Chem.217, 409-417 (2017).

21. Jin, M., Zhao, K., Huang, Q., Xu, C. \& Shang, P. Isolation, structure and bioactivities of the polysaccharides from Angelica sinensis (Oliv.) Diels: A review. Carbohydrate Polymers vol. 89 713-722 (2012).

22. Zhao, H. et al. The antihyperlipidemic activities of enzymatic and acidic intracellular polysaccharides by Termitomyces albuminosus. Carbohydr. Polym.151, 1227-1234 (2016).

23. Xie, J. H. et al. Isolation, chemical composition and antioxidant activities of a water-soluble polysaccharide from Cyclocarya paliurus (Batal.) Iljinskaja. Food Chem. (2010) doi:10.1016/j.foodchem.2009.09.055.

24. YouGuo, C., ZongJi, S. \& XiaoPing, C. Evaluation of free radicals scavenging and immunity-modulatory activities of Purslane polysaccharides. Int. J. Biol. Macromol.45, 448-452 (2009).

25. Wang, L., Liu, H. M. \& Qin, G. Y. Structure characterization and antioxidant activity of polysaccharides from Chinese quince seed meal. Food Chem. (2017) doi:10.1016/j.foodchem.2017.05.002.

26. Martin, G. M. Aging and Cell Structure. Hum. Pathol.14, 96 (1983).

27. Liu, X. et al. Physicochemical characterization of a polysaccharide from Agrocybe aegirita and its anti-ageing activity. Carbohydr. Polym. (2020) doi:10.1016/j.carbpol.2020.116056.

28. Wang, Y., Wei, X. \& Jin, Z. Structure analysis of a neutral polysaccharide isolated from green tea. Food Res. Int.42, 739-745 (2009).

29. Pannese, E. Morphological changes in nerve cells during normal aging. Brain Structure and Function vol. $21685-89$ (2011).

30. Kumar, A., Prakash, A. \& Dogra, S. Naringin alleviates cognitive impairment, mitochondrial dysfunction and oxidative stress induced by d-galactose in mice. Food Chem. Toxicol.48, 626-632 (2010).

31. Anand, K. V., Mohamed Jaabir, M. S., Thomas, P. A. \& Geraldine, P. Protective role of chrysin against oxidative stress in d-galactose-induced aging in an experimental rat model. Geriatr. Gerontol. Int.12, 741-750 (2012).

32. Lu, J. et al. Quercetin reverses d-galactose induced neurotoxicity in mouse brain. Behav. Brain Res.171, 251-260 (2006).

33. Penley, S. C., Gaudet, C. M. \& Threlkeld, S. W. Use of an eight-arm radial water maze to assess working and reference memory following neonatal brain injury. J. Vis. Exp. 50940 (2013) doi:10.3791/50940.

34. Clelland, C. D. et al. A functional role for adult hippocampal neurogenesis in spatial pattern separation. Science (80.).325, 210-213 (2009).

35. Soellner, D. E., Grandys, T. \& Nuñez, J. L. Chronic prenatal caffeine exposure impairs novel object recognition and radial arm maze behaviors in adult rats. Behav. Brain Res.205, 191-199 (2009).

36. Choi, J., Hur, T.-Y. \& Hong, Y. Influence of Altered Gut Microbiota Composition on Aging and Aging-Related Diseases. J. Lifestyle Med.8, 1-7 (2018).

37. Kelly, J. R., Minuto, C., Cryan, J. F., Clarke, G. \& Dinan, T. G. Cross talk: The microbiota and neurodevelopmental disorders. Frontiers in Neuroscience vol. 11 (2017).

38. Ahlemeyer, B. \& Krieglstein, J. Neuroprotective effects of Ginkgo biloba extract. Cellular and Molecular Life Sciences vol. 60 1779-1792 (2003).

39. Bastianetto, S. \& Quirion, R. Natural extracts as possible protective agents of brain aging. Neurobiol. Aging23, 891897 (2002).

40. Mangiola, F., Nicoletti, A., Gasbarrini, A. \& Ponziani, F. R. Gut microbiota and aging. Eur. Rev. Med. Pharmacol. Sci.22, 7404-7413 (2018).

41. Qin, J. et al. A human gut microbial gene catalogue established by metagenomic sequencing. Nature464, 59-65 (2010).

42. Carding, S., Verbeke, K., Vipond, D. T., Corfe, B. M. \& Owen, L. J. Dysbiosis of the gut microbiota in disease. Microb. Ecol. Heal. Dis. 26, (2015).

43. Saulnier, D. M. et al. The intestinal microbiome, probiotics and prebiotics in neurogastroenterology. Gut Microbes vol. 4 (2013).

44. Gareau, M. G. Cognitive Function and the Microbiome. in International Review of Neurobiology vol. $131227-246$ (Academic Press Inc., 2016).

45. Ke, R., Xu, Q., Li, C., Luo, L. \& Huang, D. Mechanisms of AMPK in the maintenance of ATP balance during energy metabolism. Cell Biology International vol. 42 384-392 (2018).

46. Sekar, P., Huang, D. Y., Hsieh, S. L., Chang, S. F. \& Lin, W. W. AMPK-dependent and independent actions of P2X7 in regulation of mitochondrial and lysosomal functions in microglia. Cell Commun. Signal.16, (2018).

47. Lee, D. H., Lee, T. H., Jung, C. H. \& Kim, Y. H. Wogonin induces apoptosis by activating the AMPK and p53 signaling pathways in human glioblastoma cells. Cell. Signal.24, 2216-2225 (2012). 
48. Yeung, F. et al. Modulation of NF-אB-dependent transcription and cell survival by the SIRT1 deacetylase. EMBO J.23, 2369-2380 (2004).

49. Salminen, A., Hyttinen, J. M. T. \& Kaarniranta, K. AMP-activated protein kinase inhibits NF- $\mathrm{BB}$ signaling and inflammation: Impact on healthspan and lifespan. Journal of Molecular Medicine vol. 89 667-676 (2011).

50. Boaru, S. G., Borkham-Kamphorst, E., Tihaa, L., Haas, U. \& Weiskirchen, R. Expression analysis of inflammasomes in experimental models of inflammatory and fibrotic liver disease. J. Inflamm. (United Kingdom) 9, (2012).

51. Shi, J. et al. Cleavage of GSDMD by inflammatory caspases determines pyroptotic cell death. Nature526, 660-665 (2015).

52. Chiurchiù, V., Orlacchio, A. \& Maccarrone, M. Is modulation of oxidative stress an answer? the state of the art of redox therapeutic actions in neurodegenerative diseases. Oxidative Medicine and Cellular Longevity vol. 2016 (2016).

53. Ou, Z. et al. Metformin treatment prevents amyloid plaque deposition and memory impairment in APP/PS1 mice. Brain. Behav. Immun.69, 351-363 (2018).

54. Li, J. et al. Mkp-1 cross-talks with Nrf2/Ho-1 pathway protecting against intestinal inflammation. Free Radic. Biol. Med.124, 541-549 (2018).

55. Huang, B. et al. $\alpha$-Cyperone inhibits LPS-induced inflammation in BV-2 cells through activation of Akt/Nrf2/HO-1 and suppression of the NF-кB pathway. Food Funct.9, 2735-2743 (2018).

56. Chen, H. H., Chen, Y. T., Huang, Y. W., Tsai, H. J. \& Kuo, C. C. 4-Ketopinoresinol, a novel naturally occurring ARE activator, induces the Nrf2/HO-1 axis and protects against oxidative stress-induced cell injury via activation of PI3K/AKT signaling. Free Radic. Biol. Med.52, 1054-1066 (2012).

57. Gao, W. et al. Glutathione homeostasis is significantly altered by quercetin via the Keap1/Nrf2 and MAPK signaling pathways in rats. J. Clin. Biochem. Nutr.62, 56-62 (2018).

58. XU, J. et al. Garcinia xanthochymus extract protects $\mathrm{PC} 12$ cells from $\mathrm{H} 2 \mathrm{O} 2$-induced apoptosis through modulation of PI3K/AKT and NRF2/HO-1 pathways. Chin. J. Nat. Med.15, 825-833 (2017).

59. Sadhukhan, P., Saha, S., Dutta, S. \& Sil, P. C. Mangiferin ameliorates cisplatin induced acute kidney injury by upregulating Nrf-2 via the activation of PI3K and exhibits synergistic anticancer activity with cisplatin. Front. Pharmacol.9, (2018).

\section{Acknowledgements}

This work was supported by the Jilin Province Science and Technology Development Projects (20191102008YY), and the Key R\&D Program of the Ministry of Science and Technology (SQ2020YFF0422819). All authors have read and approved to the published version of the manuscript.

\section{Author contributions statement}

W. L. conceived and designed the experiment, Y. Z. and X. L. conducted the experiment, Y. Z. and C. D. analysed the results. All authors reviewed the manuscript.

\section{Statement}

We confirming the study is reported in accordance with ARRIVE guidelines (https://arriveguidelines.org).

\section{Competing interests}

The authors declare that they have no competing interests.

\section{Additional information}

Correspondence requests for materials should be addressed to W. L. and C. D.

Reprints and permissions information is available at www.nature.com/reprints.

Publisher's note Springer Nature remains neutral on the regard to jurisdictional claims in the published map and institutional affiliations. 


\section{Supplementary Files}

This is a list of supplementary files associated with this preprint. Click to download.

- originalgelsorblotsfigures.docx 